\title{
Propolis from Poland versus propolis from New Zealand - chemical composition and antiproliferative properties on glioblastoma cell lines.
}

\section{Justyna Moskwa}

Uniwersytet Medyczny w Bialymstoku

\section{Sylwia Katarzyna Naliwajko}

Uniwersytet Medyczny w Bialymstoku

Renata Markiewicz-Żukowska ( $\nabla$ renmar@poczta.onet.pl)

Uniwersytet Medyczny w Bialymstoku https://orcid.org/0000-0003-0716-9573

Krystyna Joanna Gromkowska-Kępka

Uniwersytet Medyczny w Bialymstoku

\section{Katarzyna Socha}

Uniwersytet Medyczny w Bialymstoku

\section{Maria Halina Borawska}

Uniwersytet Medyczny w Bialymstoku

\section{Valery Isidorov}

Politechnika Bialostocka

\section{Research article}

Keywords: Propolis, Propolis Manuka, Glioma

Posted Date: February 12th, 2020

DOI: https://doi.org/10.21203/rs.2.23267/v1

License: (c) (1) This work is licensed under a Creative Commons Attribution 4.0 International License. Read Full License 


\section{Abstract}

Background Several studies have previously reported that propolis and its ingredients inhibit glioma cancer cell lines. The chemical composition and antiproliferative activity of propolis from Poland (PPE) and propolis from New Zealand (MPE) were compared in this study. Methods The chemical composition was investigated by gas chromatography-mass spectrometry. Antiproliferative activity of PPE and MPE was determined by a cytotoxicity test and DNA binding by [ $3 \mathrm{H}$ ]-thymidine incorporation on Human Diffuse Astrocytoma cell line (DASC) derived from a patient with a Grade II glioma and glioblastoma multiforme T98G and LN-18 cell lines from American Type Culture Collection. Results The chemical composition of both propolis was comparable, with marginal differences in the amount of some compounds. Flavonoids and chalcones, of which pinocembrin, pinobanksin, pinobanksin 3-acetate, chrysin and galangin showed the highest level, were the main components of both examined propolis (PPE-49.4\% and MPE-52.1\%). The performed cytotoxicity test showed powerful activity of PPE and MPE propolis on DASC, T98G and LN-18 cells. The degree of the antiproliferative activity was similar in the case of both propolis (viability after $72 \mathrm{~h}$ for $30 \mu \mathrm{g} / \mathrm{mL}$ ranged from $22.0 \%$ to $51.6 \%$ and proliferation inhibition after $72 \mathrm{~h}$ approximately was from $18.6 \%$ to $75.6 \%$ ). Conclusions These results are the first to show that propolis from Poland and propolis from New Zealand have a strong cytotoxic and antiproliferative effect on DASC (Grade II glioma) derived from a patient and glioblastoma multiforme T98G and LN-18 cell lines. This activity may be associated with the high content of polyphenolic compounds in both propolis. These findings suggest that Polish and New Zealand propolis shows promising anticancer activity in the treatment of glioblastoma. However, further studies are required.

\section{Background}

A number of studies have focused on the composition and properties of propolis. Propolis is a natural product composed of tree and plant resin, bee wax, pollen and gland secretions of bees. When compared to other natural products, propolis is unique since it is of both plant and animal origin. Propolis contains a wide range of active ingredients, whose concentration depends primarily on the origin, geographical provenance, season of the year and the breed of bees. There are several types of propolis: "Poplar" (European, Chinese, North and South American, including Manuka propolis from New Zealand, "Brazilian green" (containing artepillin-C), "Red" (from Cuba, Brazil, Mexico), "Brich” (from Russia), "Mediterranean" (Greece, Crete, Sicily, Malta), "Pacific" (from Okinawa, Taiwan, Indonesia) and "Clusia" (from Cuba and Venezuela) [1]. Hence, different biological activity of propolis has been reported by different authors. The most active compounds are flavonoids (e.g. chrysin, apigenin, pinocembrin, pinobanksin, kaempferol), aromatic acids (e.g. p-coumaric, ferulic) and esters (caffeic acid phenethyl ester - CAPE) [2, 3]. A number of studies concerning the anti-cancer activity of propolis on various cancer cell lines such as human colorectal cancer (DLD-1) [1], human lung cancer (A549) [4], gastric cancer (HGC27) [5] and human prostate cancer (PC3) [6] have been published. The antiproliferative potential of propolis from Poland on the human glioblastoma multiforme cell line U87MG has been confirmed in our previous studies $[2,7,8]$. 
The present study is the first to compare the chemical composition and antiproliferative activity of propolis from Poland and propolis from New Zealand on Human Diffuse Astrocytoma cell lines (DASC) derived from a patient with Grade II glioma and glioblastoma multiforme T98G and LN-18 cell lines.

\section{Methods}

\section{Materials}

DMEM/Ham's F12 with L-glutamine was purchased from PAA Laboratories GmbH (Pasching, Austria). Dulbecco's modified eagle medium (DMEM), fetal bovine serum (FBS), minimal essential medium eagle (MEM) with L-glutamine, trypsin-EDTA, penicillin, streptomycin were purchased from Gibco (Thermo Fisher Scientific, Waltham, USA). Calcium-free phosphate buffered saline (PBS) was received from Biomed (Lublin, Poland). Bis(trimethylsilyl)trifluoroacetamide (BSTFA) with an addition of $1 \%$ trimethylchlorosilane, $\mathrm{C}_{10}-\mathrm{C}_{40}$ n-alkane standard solution, methylthiazolyl diphenyl-tetrazolium bromide (MTT), dimethyl sulfoxide (DMSO), pyridine, trichloroacetic acid, trizma base were obtained from SigmaAldrich (St. Louis, USA). Ethanol 95\% (AWW Group, Poland). The scintillation cocktail was purchased from PerkinElmer (Boston, MA). Methyl-3H thymidine from MP Biomedicals, Inc. (Irvine, USA).

\section{Sample preparations}

Propolis of Apis mellifera was collected in the Podlasie region (northeastern Poland). To prepare the ethanolic extract of Polish propolis (PPE), $20 \mathrm{~g}$ of crushed propolis was extracted on a shaker with $80 \mathrm{~g}$ of $70 \%$ ethanol for $12 \mathrm{~h}$ in a darkened place. The extract was centrifuged at $2500 \mathrm{rpm}$ for $10 \mathrm{~min}$ at $20^{\circ} \mathrm{C}$, evaporated $\left(40^{\circ} \mathrm{C}\right)$ in a rotary evaporator (Rotavapor R-3, Buchi, Switzerland) and lyophilised. The dry Polish propolis extract (PPE) was protected from light and kept frozen at $-20^{\circ} \mathrm{C}$. The yield of the prepared extracts $(\% \mathrm{w} / \mathrm{w})$ in terms of the starting material was 47.6 .

Propolis Manuka Health New Zealand (Bio 30) ethanolic tincture was purchased from the manufacturer. The tincture was evaporated $\left(40^{\circ} \mathrm{C}\right)$ in a rotary evaporator (Rotavapor R-3, Buchi, Switzerland) and lyophilised. The dry Manuka Propolis extract (MPE) was protected from light and kept frozen at $-20^{\circ} \mathrm{C}$.

The extracts were dissolved in DMSO and prepared as $1 \mathrm{mg} / \mathrm{mL}$ stock solution (calculated as dry extracts) in the culture medium.

\section{Gass chromatography-mass spectrometry (GC-MS) analysis}

$5 \mathrm{mg}$ of PPE and MPE were diluted with $220 \mu \mathrm{L}$ of pyridine and $80 \mu \mathrm{L}$ of BSTFA with an addition of $1 \%$ trimethylchlorosilane. The reaction mixture was sealed and heated for $0.5 \mathrm{~h}$ at $60{ }^{\circ} \mathrm{C}$ to form trimethylsilyl (TMS) derivatives.

GC-MS analyses of PPE and MPE were performed using GC-MS on a HP 6890 gas chromatograph with a mass selective detector MSD 5973 (Agilent Technologies, USA) equipped with a ZB-5MSi fused silica column ( $30 \mathrm{~m}, 0.25 \mathrm{~mm}$ i.d., $0.25 \mu \mathrm{m}$ film thickness), with electronic pressure control and a split/splitless 
injector. Helium flow rate through the column was $1 \mathrm{~mL} / \mathrm{min}$ in a constant flow mode. The injector worked at $250^{\circ} \mathrm{C}$ in the split $(1: 50)$ mode. The initial column temperature was $50^{\circ} \mathrm{C}$, rising to $310^{\circ} \mathrm{C}$ at $5^{\circ} \mathrm{C} / \mathrm{min}$ and the higher temperature was maintained for $15 \mathrm{~min}$. MSD detector acquisition parameters were as follows: transfer line temperature $280^{\circ} \mathrm{C}$, MS Source temperature $230^{\circ} \mathrm{C}$ and MS Quad temperature $150^{\circ} \mathrm{C}$. The EIMS spectra were obtained at the ionisation energy of $70 \mathrm{eV}$. The MSD was set to scan 41-600 a.m.u. Following the integration, the fraction of each component in the total ion current was calculated. Hexane solutions of $\mathrm{C}_{10}-\mathrm{C}_{40}$ n-alkanes were separated under the above conditions. Gas chromatographic linear programmed retention indices $\left(I_{T}\right)$ were calculated on the basis of the retention times of the n-alkanes hexane solution and separated components of the extract samples.

To identify the separated components, two independent analytical parameters were used: mass spectra and calculated retention indices. The mass spectrometric identification of non-derivatised components was performed with an automatic system for GC-MS data processing supplied by the NIST 14 library (NIST/EPA/NIH Library of Electron Ionization Mass Spectra). The mass spectra and retention indices of the components registered in the form of TMS derivatives were compared with those presented in a recently published database [9] and a private mass spectra library. Identification was considered reliable if the results of the computer search of the mass spectra library were confirmed by experimental RI values, i.e. if their deviation from the published database values did not exceed \pm 10 u.i. (the average quantity of inter-laboratory deviation for non-polar stationary phases).

\section{Total phenolic content analysis}

Total phenolic content (TPC) was measured using the Folin-Ciocalteu colorimetric method (FC). Absorbance versus a prepared blank was read at $760 \mathrm{~nm}$ using Cintra 3030 (GBC Scientific Equipment, Australia). The results were expressed as milligrams of gallic acid equivalent (GAE) per gram of a dry extract. The concentration of samples equalled $2 \mathrm{mg} / \mathrm{mL}$ (extract dissolved in $70 \%$ ethanol). Assays were performed in triplicate. Data were expressed as mean \pm SD.

\section{Cell culture}

The study was performed using Diffuse astrocytoma steam-like cells (DASC) and glioblastoma multiforme (T98G and LN-18) cell lines. DASC cell line was derived from a 43-year-old patient with diffuse astrocytoma (Grade II), which was described in our previous research [10]. The study was approved by the local Ethics Committee [10]. T98G and LN-18 were obtained from American Type Culture Collection (ATCC, Rockville, MD, USA). The cells were cultured in a humidified incubator at $37^{\circ} \mathrm{C}$ and $5 \% \mathrm{CO}_{2}$ atmosphere, in MEM (DASC and T98G) or DMEM (LN-18) supplemented with $10 \%$ heat inactivated FBS; $100 \mathrm{U} / \mathrm{mL}$ penicillin and $0.1 \mathrm{mg} / \mathrm{mL}$ streptomycin. Subconfluent cells were detached with a trypsin-EDTA solution in PBS and counted in a Neubauer hemocytometer.

\section{Cytotoxicity assay}

Cell viability was measured using an MTT assay, as previously described for glioma cells [10]. The effects of PPE and MPE extracts on DASC, T98G and LN-18 cell lines were studied after $24 \mathrm{~h}, 48 \mathrm{~h}$ and 
$72 \mathrm{~h}$ of the treatment. The cells were cultured in a humidified incubator at $37^{\circ} \mathrm{C}$ and $5 \% \mathrm{CO}_{2}$ atmosphere; in MEM or DMEM supplemented with $10 \%$ heat inactivated FBS; $100 \mathrm{U} / \mathrm{mL}$ penicillin and $0.1 \mathrm{mg} / \mathrm{mL}$ streptomycin. Doses of propolis $(10,20,30,50,100 \mu \mathrm{g} / \mathrm{mL})$ were selected in our previous experiments [8]. Cells at a density of $1 \times 10^{5}$ cells $/ \mathrm{mL}$ were seeded onto 96 -well plates at a volume of $200 \mu \mathrm{l}$ per well and grown for $22 \mathrm{~h}$ at $37^{\circ} \mathrm{C}$ in a humidified $5 \% \mathrm{CO}_{2}$ incubator. The data were expressed as a percentage of the control (0.1\% DMSO).

\section{DNA synthesis assay}

$\left[{ }^{3} \mathrm{H}\right]$-thymidine assays were performed to study DNA synthesis in the cells after the treatment, as described in our previously published study [10]. The cells were seeded $\left(1.5 \times 10^{5}\right.$ cell/well) on 24-well plates in MEM or DMEM supplemented with $10 \%$ heat inactivated FBS; $100 \mathrm{U} / \mathrm{mL}$ penicillin and $0.1 \mathrm{mg} / \mathrm{mL}$ streptomycin and exposed to the treatment medium containing DMSO (0.1\% - control), PPE and MPE $(30 \mu \mathrm{g} / \mathrm{mL})$. The cells were cultured for $44 \mathrm{~h}$ prior to adding $0.5 \mu \mathrm{Ci}$ of ${ }^{3} \mathrm{H}$-thymidine per well. After $4 \mathrm{~h}$ of incubation, the medium was removed and the cells were washed twice with cold $0.05 \mathrm{M}$ Tris$\mathrm{HCl}$ and $5 \%$ trichloroacetic acid, then scraped and transferred to a scintillation cocktail. The level of $\left[{ }^{3} \mathrm{H}\right]-$ thymidine incorporated in the newly synthesised DNA strand was assessed by a scintillation counter in relation to the number of cells proliferating during the $S$ phase of the cell cycle.

\section{Statistical analysis}

All data were analysed using Dell Inc. (2016). Dell Statistica (data analysis software system), version 13. software.dell.com. The results were expressed as mean \pm SD and statistically compared to the control. Values were tested for a normal distribution using the Shaphiro-Wilk test. Differences between two groups were analyzed using Student's t-test or U Mann-Whitney test. $\mathrm{P}<0.05$ was considered to be statistically significant.

\section{Results And Discussion}

\section{Chemical composition of Polish and Manuka propolis}

The complex chemical composition of propolis is associated with the quality of the resinous materials gathered by honey bees from different floral sources available around the hive, which has a direct impact on the quality and bioactivity of propolis. In this study, more than 100 individual compounds in PPE and more than 150 compounds in MPE were identified. A list of these constituents is presented in Table 1. Flavonoids and chalcones were the main components of both examined propolis (PPE- $49.4 \%$ and MPE$52.1 \%$ ) (Table 2). The main representatives of this group of compounds in PPE and MPE were, respectively, pinocembrin (8.16\% and $14.64 \%)$, pinobanksin (4.25\% and $4.70 \%)$, pinobanksin 3-acetate $(11.27 \%$ and $9.21 \%)$, chrysin $(5.33 \%$ and $5.73 \%)$, galangin $(8.95 \%$ and $9.60 \%)$ and their derivatives. (Table 1). These compounds are characteristic of propolis originating from bud exudates of Populus nigra $[11,3]$. Our analysis also confirmed research results published by other authors who have demonstrated that New Zealand propolis has very high levels of pinocembrin and pinobanksin-3-0- 
acetate [1]. Cinnamic acid derivatives such as esters: 3-methyl-2-bytenyl (E)-caffeate, benzyl (E)-caffeate, benzyl (E)-p-coumarate, 2-phenylethyl p-coumarate, benzyl (E)-ferulate, CAPE, cinnamyl (E)-p-coumarate and others were the second significant group of compounds in PPE and MPE (19.8\% and 14.5\%) (Table 2). Considerable quantities of aromatic acids were present in both studied propolis extracts, although propolis from Poland (PPE-18.3\%) contained twice as great a quantity of aromatic acids as propolis from New Zealand (MPE-7.8\%) (Table 2). The main representatives of this group were pcoumaric acid, (E)-ferulic acid and (E)-caffeic acid. PPE contained high levels of p-coumaric acid (9.80\%) (Table 1). TPC determination confirmed that PPE and MPE are rich in phenolic compounds. Their levels were calculated to be $243.7 \pm 9.0$ in PPE and $245.6 \pm 5.9 \mathrm{mg} \mathrm{GAE} / \mathrm{g}$ in MPE (Table 3). Other authors have demonstrated higher or lower TPC in propolis. The values ranged from 14.6 to $150.8 \mathrm{mg} \mathrm{GAE} / \mathrm{g}$ in Polish propolis [12] and from $99 \pm 4.0$ to $775 \pm 8.5 \mathrm{mg} \mathrm{GAE} / \mathrm{g}$ in Manuka propolis [13]. TPC value depended on the extraction method utilised.

Comparison of the chemical composition of the tested propolis revealed that both PPE and MPE had similar quantities of the identified active components and the total content of phenols, which is consistent with the classification of propolis from New Zealand as the "Poplar" type. According to Kumazawa et al. [14], comparison of the antioxidant activity and composition, total phenol and flavonoid content in individual samples of ethanolic extracted propolis from 14 countries showed that New Zealand-sourced propolis was similar in composition to propolis from Bulgaria, Uzbekistan and Hungary, and to propolis from three South American countries: Chile, Uruguay and Argentina. 
Table 1

Chemical composition of the ethanolic extracts of propolis from Poland (PPE) and New Zealand (MPE)

\begin{tabular}{|c|c|c|c|c|c|}
\hline Components & CAS & $\mathrm{I}_{\mathrm{T}}^{\operatorname{Exp}}$ & $\mathrm{I}_{\mathrm{T}}^{\mathrm{Lit}}$ & $\begin{array}{l}\text { PPE } \\
\text { [\%] }\end{array}$ & $\begin{array}{l}\text { MPE } \\
\text { [\%] }\end{array}$ \\
\hline Benzyl alcohol, mono-TMS & $\begin{array}{l}14642-79- \\
6\end{array}$ & 1152 & 1155 & trace* & 0.09 \\
\hline 2-Phenyl ethanol, mono-TMS & $\begin{array}{l}14629-58- \\
4\end{array}$ & 1225 & 1227 & trace & 0.04 \\
\hline Benzoic acid, mono-TMS & $2078-12-8$ & 1244 & 1247 & 1.80 & 0.33 \\
\hline Diethylene glycol, di-TMS? $(73,117,103,147,191)$ & $\begin{array}{l}16654-74- \\
3\end{array}$ & 1250 & 1239 & - & 0.07 \\
\hline $\mathrm{H}_{3} \mathrm{PO}_{4}$, tri-TMS & $\begin{array}{l}10497-05- \\
9\end{array}$ & 1289 & 1289 & - & 0.02 \\
\hline Glycerol, tri-TMS & $6787-10-6$ & 1291 & 1293 & 0.22 & 0.57 \\
\hline Succinic acid, di-TMS & $\begin{array}{l}40309-57- \\
7\end{array}$ & 1321 & 1324 & - & trace \\
\hline Ethyl dihydrocinnamate & $2021-28-5$ & 1345 & 1349 & - & trace \\
\hline Hydroquinone, di-TMS & $2117-24-0$ & 1406 & 1410 & 0.04 & trace \\
\hline 3-Hydroxy acid, di-TMS? $(147,73,103,233)$ & - & 1410 & - & - & trace \\
\hline Hydrocinnamic acid, mono-TMS & $\begin{array}{l}21273-15- \\
4\end{array}$ & 1414 & 1418 & trace & 0.06 \\
\hline Cinnamoyl alcohol, mono-TMS & $N / A^{\star \star}$ & 1425 & 1427 & trace & 0.03 \\
\hline 3-Hydroxy acid, di-TMS? $(73,131,147,233)$ & - & 1461 & - & - & 0.02 \\
\hline 4'-Hydroxyacetophenone, mono-TMS & $\begin{array}{l}18803-29- \\
7\end{array}$ & 1467 & 1471 & - & trace \\
\hline Malic acid, tri-TMS & $\begin{array}{l}38166-11- \\
9\end{array}$ & 1507 & 1511 & - & 0.03 \\
\hline Vanillin, mono-TMS & $6689-43-6$ & 1534 & 1533 & trace & trace \\
\hline Erythritol, tetra-TMS & $\begin{array}{l}25258-02- \\
0\end{array}$ & 1536 & 1535 & - & 0.06 \\
\hline Cinnamic acid, mono-TMS & $2078-20-8$ & 1542 & 1546 & 0.20 & 1.82 \\
\hline 7-Phenyl-5-hepten-2-one? $(130,129,91)$ & $\begin{array}{l}33046-89- \\
6\end{array}$ & 1567 & - & - & 0.03 \\
\hline Protocatechuic aldehyde, di-TMS & $\mathrm{N} / \mathrm{A}$ & 1619 & 1620 & trace & 0.07 \\
\hline
\end{tabular}




\begin{tabular}{|c|c|c|c|c|c|}
\hline Components & CAS & $\mathrm{I}_{\mathrm{T}}^{\operatorname{Exp}}$ & $\mathrm{I}_{\mathrm{T}}^{\mathrm{Lit}}$ & $\begin{array}{l}\text { PPE } \\
{[\%]}\end{array}$ & $\begin{array}{l}\text { MPE } \\
{[\%]}\end{array}$ \\
\hline NN $(73>130,75,233,248)$ & - & 1626 & - & - & 0.15 \\
\hline 4-Hydroxybenzoic acid, di-TMS & $2078-13-9$ & 1632 & 1636 & 0.16 & 0.03 \\
\hline Docosanoic acid, mono-TMS & $\begin{array}{l}55520-95- \\
1\end{array}$ & 1661 & 1658 & trace & - \\
\hline Guaiol, mono-TMS & $\mathrm{N} / \mathrm{A}$ & 1682 & 1685 & trace- & 0.07 \\
\hline Acorenol, mono-TMS & $\mathrm{N} / \mathrm{A}$ & 1720 & 1723 & - & 0.02 \\
\hline Agarospirol, mono-TMS & N/A & 1737 & 1734 & - & 0.01 \\
\hline Y-Eudesmol, mono-TMS & N/A & 1740 & 1739 & - & 0.11 \\
\hline a-Bisabolol, mono-TMS & N/A & 1747 & 1752 & trace & 0.05 \\
\hline$\beta$-Eudesmol, mono-TMS & N/A N/A & 1749 & 1751 & - & 0.09 \\
\hline Arabinitol, penta-TMS & $\begin{array}{l}25138-28- \\
7\end{array}$ & 1755 & 1760 & - & 0.07 \\
\hline Benzyl benzoate & $120-51-4$ & 1764 & 1763 & 0.29 & - \\
\hline Vanillic acid, di-TMS & $2078-15-1$ & 1776 & 1776 & trace & - \\
\hline (Z)-p-Coumaric acid, di-TMS & N/A & 1799 & 1798 & 0.12 & - \\
\hline Methylfuranoside, tetra-TMS & $\begin{array}{l}30788-71- \\
7\end{array}$ & 1811 & 1813 & - & 0.11 \\
\hline p-Methoxycinnamic acid, mono-TMS & $\begin{array}{l}25436-23- \\
1\end{array}$ & 1827 & 1830 & - & 0.11 \\
\hline Cinnamylideneacetic acid, mono-TMS & N/A & 1835 & 1840 & trace & 0.63 \\
\hline a-Fructofuranose, penta-TMS & N/A & 1841 & 1845 & 0.26 & 0.69 \\
\hline$\beta$-Fructofuranose, penta-TMS & $\mathrm{N} / \mathrm{A}$ & 1850 & 1854 & 3.67 & 7.32 \\
\hline a-Mannofuranose, penta-TMS & $\mathrm{N} / \mathrm{A}$ & 1872 & 1874 & - & 0.35 \\
\hline a-Glucofuranose, penta-TMS & $\begin{array}{l}66807-66- \\
7\end{array}$ & 1884 & 1885 & - & 0.11 \\
\hline NN $(73,131,204,368,203)$ & - & 1896 & - & - & 0.06 \\
\hline a-Glucopyranose, penta-TMS & $\mathrm{N} / \mathrm{A}$ & 1929 & 1930 & 0.91 & 4.02 \\
\hline p-Coumaric acid, di-TMS & $\begin{array}{l}10517-30- \\
3\end{array}$ & 1944 & 1947 & 9.77 & 0.87 \\
\hline
\end{tabular}

* trace - below $0.01 \%$ of the total ion current. ** N/A - not available 


\begin{tabular}{|c|c|c|c|c|c|}
\hline Components & CAS & $\mathrm{I}_{\mathrm{T}}^{\operatorname{Exp}}$ & $\mathrm{I}_{\mathrm{T}}^{\mathrm{Lit}}$ & $\begin{array}{l}\text { PPE } \\
\text { [\%] }\end{array}$ & $\begin{array}{l}\text { MPE } \\
\text { [\%] }\end{array}$ \\
\hline $\begin{array}{l}\text { Sesquiterpenol C15H260-TMS? (131 > 73... } \\
279,103)\end{array}$ & - & 1948 & - & - & 0.26 \\
\hline NN $(131,73,249,179 \ldots 399,355)$ & - & 1956 & - & - & 0.04 \\
\hline Mannitol, hexa-TMS & $\begin{array}{l}14317-07- \\
8\end{array}$ & 1970 & 1972 & - & 0.04 \\
\hline Sedoheptulose, hexa-TMS & $\begin{array}{l}\text { 74987-26- } \\
0\end{array}$ & 1974 & 1972 & - & 0.16 \\
\hline Ethyl hexadecanoate & $628-97-7$ & 1990 & 1994 & - & 0.03 \\
\hline NN $(73,147,289,248,319 \ldots 379)$ & - & 2007 & - & - & 0.05 \\
\hline $\beta$-Glucopyranose, penta-TMS & $2775-90-8$ & 2028 & 2032 & 0.99 & 5.25 \\
\hline 3,4-Dimethoxycinnamic acid, mono-TMS & $\begin{array}{l}27750-71- \\
6\end{array}$ & 2030 & 2034 & - & 1.51 \\
\hline Gluconic acid, hexa-TMS & $\begin{array}{l}34290-52- \\
3\end{array}$ & 2041 & 2045 & - & 0.04 \\
\hline Hexadecanoic acid, mono-TMS & $\begin{array}{l}55520-89- \\
3\end{array}$ & 2049 & 2052 & 0.27 & 0.11 \\
\hline (E)-1,4-Diphenyl-3-buten-1-one & $\begin{array}{l}32363-55- \\
6\end{array}$ & 2072 & - & - & trace \\
\hline Isoferulic acid, di-TMS & $\begin{array}{l}32342-04- \\
4\end{array}$ & 2087 & 2088 & 0.95 & 0.82 \\
\hline Ethyl caffeate, di-TMS & N/A & 2092 & 2091 & - & 0.04 \\
\hline NN $(131,73>162,143)$ & - & 2096 & - & - & 0.26 \\
\hline (E)-Ferulic acid, di-TM & $\begin{array}{l}10517-09- \\
6\end{array}$ & 2101 & 2101 & 3.22 & 0.15 \\
\hline myo-Inositol, hexa-TMS & $2582-79-8$ & 2124 & 2125 & trace & 0.04 \\
\hline NN $(73$ > 157,156) & - & 2148 & - & - & 0.06 \\
\hline 3-Methylbutanyl (E)-p-coumarate, mono-TMS & N/A & 2152 & 2145 & 0.65 & - \\
\hline (E)-Caffeic acid, di-TMS & $\begin{array}{l}10586-03- \\
5\end{array}$ & 2155 & 2155 & 2.10 & 1.53 \\
\hline 3-Methyl-3-butenyl p-coumarate, mono-TMS & N/A & 2159 & 2159 & 0.18 & 0.11 \\
\hline $\mathrm{NN}(247>73,131 \ldots 358)$ & - & 2169 & - & - & 0.10 \\
\hline
\end{tabular}

* trace - below $0.01 \%$ of the total ion current. ** N/A - not available 


\begin{tabular}{|c|c|c|c|c|c|}
\hline Components & CAS & $\mathrm{I}_{\mathrm{T}}^{\operatorname{Exp}}$ & $\mathrm{I}_{\mathrm{T}}^{\mathrm{Lit}}$ & $\begin{array}{l}\text { PPE } \\
\text { [\%] }\end{array}$ & $\begin{array}{l}\text { MPE } \\
\text { [\%] }\end{array}$ \\
\hline 2-Methyl-2-butenyl p-coumarate, mono-TMS & N/A & 2205 & 2201 & 0.97 & trace \\
\hline 3-Methyl-2-butenyl p-coumarate, mono-TMS & N/A & 2212 & 2216 & 0.23 & 0.07 \\
\hline Linoleic acid, mono-TMS & $\begin{array}{l}56259-07- \\
5\end{array}$ & 2217 & 2215 & trace & 0.03 \\
\hline Oleic acid, mono-TMS & $\begin{array}{l}21556-26- \\
3\end{array}$ & 2222 & 2222 & 0.33 & 0.21 \\
\hline NN $(73>156,244,143,93,147 \ldots 381)$ & - & 2234 & - & 1.31 & 0.17 \\
\hline Octadecanoic acid, mono-TMS & $\begin{array}{l}18748-91- \\
9\end{array}$ & 2249 & 2252 & trace & 0.06 \\
\hline 3-Methyl-3-butenyl isoferulate, mono-TMS & N/A & 2303 & 2304 & - & 0.38 \\
\hline 3-Methyl-3-butenyl (E)-ferulate, mono-TMS & N/A & 2318 & 2319 & 0.07 & 0.16 \\
\hline Benzyl (Z)-p-coumarate, mono-TMS & N/A & 2323 & 2329 & 0.07 & - \\
\hline Eicosanoic acid, mono-TMS & $\begin{array}{l}55530-70- \\
6\end{array}$ & 2349 & 2349 & - & 0.03 \\
\hline NN $(335,73,446,147,69,41,147)$ & - & 2346 & - & - & 0.04 \\
\hline 3-Methylbutanyl (E)-caffeate, di-TMS & N/A & 2358 & 2358 & 0.06 & 0.15 \\
\hline 3-Methyl-2-butenyl (E)-isoferulate, mono-TMS & N/A & 2365 & 2365 & - & 0.05 \\
\hline 3-Methyl-3-butenyl (E)-caffeate, di-TMS & N/A & 2371 & 2367 & 1.18 & 3.39 \\
\hline 3-Methyl-2-butenyl (E)-caffeate, di-TMS & N/A & 2374 & 2375 & 0.50 & 0.44 \\
\hline NN $(397,369,73,91)$ & - & 2384 & - & - & 0.03 \\
\hline Pinostrobin chalcone & $\begin{array}{l}18956-15- \\
5\end{array}$ & 2392 & - & 0.16 & 0.07 \\
\hline NN $(73,75,55,143,207,129,41)$ & - & 2404 & - & - & 0.02 \\
\hline Cinnamyl cinnamate & $122-69-0$ & 2408 & 2391 & - & 0.29 \\
\hline 2-Methyl-2-butenyl (E)-caffeate, di-TMS & N/A & 2414 & 2413 & 0.09 & 0.24 \\
\hline $\begin{array}{l}\text { 2',6'-Dihydroxy-4'-methoxydihydrochalcone, di- } \\
\text { T'MS }\end{array}$ & $\mathrm{N} / \mathrm{A}$ & 2418 & 2416 & 0.46 & 0.02 \\
\hline 3-Methyl-2-bytenyl (E)-caffeate, di-TMS & N/A & 2425 & 2421 & 1.65 & 2.36 \\
\hline NN $(143,73,81,95,121,151)$ & - & 2444 & - & - & 0.02 \\
\hline
\end{tabular}

* trace - below $0.01 \%$ of the total ion current. ${ }^{* *} \mathrm{~N} / \mathrm{A}$ - not available 


\begin{tabular}{|c|c|c|c|c|c|}
\hline Components & CAS & $\mathrm{I}_{\mathrm{T}}^{\operatorname{Exp}}$ & $\mathrm{I}_{\mathrm{T}}^{\mathrm{Lit}}$ & $\begin{array}{l}\text { PPE } \\
\text { [\%] }\end{array}$ & $\begin{array}{l}\text { MPE } \\
\text { [\%] }\end{array}$ \\
\hline NN $(287,372,357,263,73)$ & - & 2450 & - & - & 0.18 \\
\hline NN $(262,73,247,460,375,287,445)$ & - & 2452 & - & - & 0.25 \\
\hline Pinocembrin, mono-TMS & $\mathrm{N} / \mathrm{A}$ & 2460 & 2461 & 1.14 & 0.46 \\
\hline 2',6',a-Trihydroxy-4'-methoxychalcone, tri-TMS & $\mathrm{N} / \mathrm{A}$ & 2491 & 2492 & 0.14 & - \\
\hline (Z)-Coniferyl benzoate, mono-TMS & $\mathrm{N} / \mathrm{A}$ & 2494 & 2495 & - & 0.15 \\
\hline n-Pentacosane & $629-99-2$ & 2500 & 2500 & trace & trace \\
\hline Pinostrobin chalcone, di-TMS & $\mathrm{N} / \mathrm{A}$ & 2506 & 2508 & 0.26 & 0.04 \\
\hline Pinostrobin, mono-TMS & $\mathrm{N} / \mathrm{A}$ & 2512 & 2512 & 0.66 & 0.84 \\
\hline Benzyl (E)-p-coumarate, mono-TMS & $\mathrm{N} / \mathrm{A}$ & 2516 & 2515 & 3.78 & 0.37 \\
\hline 1-p-Coumaroyl glycerol, tri-TMS & $\mathrm{N} / \mathrm{A}$ & 2528 & 2528 & 0.06 & - \\
\hline Pinocembrin chalcone, tri-TMS & $\mathrm{N} / \mathrm{A}$ & 2542 & 2541 & 0.09 & 0.08 \\
\hline Pinocembrin, di-TMS & $\mathrm{N} / \mathrm{A}$ & 2551 & 2552 & 6.93 & 14.10 \\
\hline NN $(73,75,121,81,95,143 \ldots)$ & - & 2555 & - & - & 0.05 \\
\hline NN $(303>73,95,147,213,225)$ & - & 2563 & - & - & 0.81 \\
\hline NN $(262,73,247,460,375)$ & - & 2569 & - & - & 0.09 \\
\hline 2-Acetyl-1-p-coumaroyl glycerol, di-TMS & $\mathrm{N} / \mathrm{A}$ & 2578 & 2578 & 0.12 & - \\
\hline 1-Acetyl-3-p-coumaroyl glycerol, di-TMS & $\mathrm{N} / \mathrm{A}$ & 2581 & 2580 & 0.19 & - \\
\hline Chalcone, TMS? $(192,73,311,238)$ & $\mathrm{N} / \mathrm{A}$ & 2586 & - & trace & 0.26 \\
\hline 2-Phenylethyl p-coumarate, mono-TMS & $\mathrm{N} / \mathrm{A}$ & 2603 & 2603 & 1.02 & 0.11 \\
\hline Pinobanksin, tri-TMS & $\mathrm{N} / \mathrm{A}$ & 2613 & 2611 & 4.25 & 4.73 \\
\hline 3-Hydroxyeicosanoic acid, di-TMS & $\mathrm{N} / \mathrm{A}$ & 2623 & 2620 & - & 0.03 \\
\hline Pinobanksin 3-acetate, mono-TMS & $\mathrm{N} / \mathrm{A}$ & 2634 & 2632 & 1.26 & 0.21 \\
\hline Coniferyl benzoate, mono-TMS & $\mathrm{N} / \mathrm{A}$ & 2637 & 2640 & trace & - \\
\hline Chrysin, mono-TMS & $\mathrm{N} / \mathrm{A}$ & 2655 & 2648 & 1.95 & 0.42 \\
\hline Benzyl (E)-isoferulate, mono-TMS & $\mathrm{N} / \mathrm{A}$ & 2659 & 2659 & - & 0.26 \\
\hline 2',6'-Dihydroxy-4,4'-dimethoxydihydrochalcone & $\mathrm{N} / \mathrm{A}$ & 2659 & 2659 & trace & \\
\hline
\end{tabular}




\begin{tabular}{|c|c|c|c|c|c|}
\hline Components & CAS & $\mathrm{I}_{\mathrm{T}}^{\operatorname{Exp}}$ & $\mathrm{I}_{\mathrm{T}}^{\mathrm{Lit}}$ & $\begin{array}{l}\text { PPE } \\
\text { [\%] }\end{array}$ & $\begin{array}{l}\text { MPE } \\
\text { [\%] }\end{array}$ \\
\hline NN $(238,385,325,73,43,341)$ & - & 2666 & - & 0.34 & 0.21 \\
\hline Pinobanksin x-acetate, TMS? $(296,443,73,383)$ & - & 2671 & - & - & 0.58 \\
\hline 5,7-Dihydroxy-3-methoxyflavanone & N/A & 2675 & 2673 & 2.02 & 2.04 \\
\hline Benzyl (E)-ferulate, mono-TMS & $\mathrm{N} / \mathrm{A}$ & 2680 & 2680 & 1.64 & 0.45 \\
\hline Pinobanksin 3-acetate, di-TMS & N/A & 2694 & 2693 & 10.01 & 9.00 \\
\hline NN (325 >> 282,155,73) & - & 2706 & - & 0.14 & 0.85 \\
\hline Sucrose, octa-TMS & $\begin{array}{l}19159-25- \\
2\end{array}$ & 2714 & 2714 & 0.25 & 0.33 \\
\hline Galangin, di-TMS & N/A & 2719 & 2717 & trace & trace \\
\hline Benzyl (E)-caffeate, di-TMS & N/A & 2723 & 2722 & 3.79 & 2.70 \\
\hline $\begin{array}{l}\text { 2',6',4-Trihydroxy-4'-methoxydihydrochalcone, tri- } \\
\text { T'MS }\end{array}$ & $\mathrm{N} / \mathrm{A}$ & 2636 & 2637 & 0.14 & - \\
\hline Pinobanksin 3-propanoate, di-TMS & N/A & 2737 & - & - & 0.06 \\
\hline Isosakuranetin, mono-TMS & N/A & 2740 & 2742 & trace & - \\
\hline Chrysin, di-TMS & N/A & 2746 & 2745 & 5.33 & 5.73 \\
\hline 5,7-Dihydroxy-3-methoxyflavone, di-TMS & N/A & 2755 & 2750 & 0.67 & 0.60 \\
\hline 1-Acetyl-3-caffeoyl glycerol, tri-TMS & N/A & 2761 & 2768 & 0.06 & - \\
\hline Galangin, tri-TMS & N/A & 2767 & 2769 & 8.95 & 9.60 \\
\hline Disaccharide, TMS & - & 2775 & - & - & 0.12 \\
\hline Pinobanksin 3-isobutanoate, di-TMS & $\mathrm{N} / \mathrm{A}$ & 2788 & 2791 & 0.52 & 0.51 \\
\hline$\beta$-Maltose, octa-TMS & N/A & 2797 & 2800 & 0.07 & 0.14 \\
\hline CAPE, di-TMS & N/A & 2805 & 2805 & 1.29 & 1.15 \\
\hline Isosakuranetin, di-TMS + disaccharide & - & 2816 & 2820 & 1.34 & - \\
\hline Isosakuranetin, di-TMS & N/A & 2816 & 2820 & - & 0.18 \\
\hline Dihydroxymethoxyflavone, di-TMS & - & 2821 & 2820 & 0.39 & 0.40 \\
\hline Cinnamyl (E)-p-coumarate, mono-TMS & $\mathrm{N} / \mathrm{A}$ & 2836 & 2833 & 1.91 & 0.23 \\
\hline
\end{tabular}

* trace - below $0.01 \%$ of the total ion current. ** N/A - not available 


\begin{tabular}{|c|c|c|c|c|c|}
\hline Components & CAS & $\mathrm{I}_{\mathrm{T}}^{\operatorname{Exp}}$ & $\mathrm{I}_{\mathrm{T}}^{\mathrm{Lit}}$ & $\begin{array}{l}\text { PPE } \\
\text { [\%] }\end{array}$ & $\begin{array}{l}\text { MPE } \\
\text { [\%] }\end{array}$ \\
\hline Tetracosanoic acid, mono-TMS & $\begin{array}{l}74367-37- \\
6\end{array}$ & 2844 & 2845 & 0.53 & - \\
\hline Pinobanksin-3-n-butanoate, di-TMS & $\mathrm{N} / \mathrm{A}$ & 2848 & 2849 & 0.17 & 0.13 \\
\hline Disaccharide, TMS $(73,361,217)$ & - & 2857 & - & - & 0.09 \\
\hline Sakuranetin chalcone, tri-TMS & $\mathrm{N} / \mathrm{A}$ & 2871 & 2871 & - & 0.05 \\
\hline Sakuranetin, di-TMS & $\mathrm{N} / \mathrm{A}$ & 2877 & 2880 & 0.55 & 0.05 \\
\hline Pinobanksin 5-pentanoate, di-TMS & $\mathrm{N} / \mathrm{A}$ & 2885 & 2884 & 0.19 & 0.58 \\
\hline$\beta$-Cellobiose, octa-TMS & $\mathrm{N} / \mathrm{A}$ & 2889 & 2888 & - & 0.12 \\
\hline Naringenin, tri-TMS & $\mathrm{N} / \mathrm{A}$ & 2895 & 2895 & 0.23 & 0.06 \\
\hline NN $(191,117,91)$ & - & 2933 & - & - & 0.03 \\
\hline Disaccharide, TMS $(204,73,361)$ & - & 2956 & - & - & 0.03 \\
\hline Pinobanksin 5-pentenoate, di-TMS & $\mathrm{N} / \mathrm{A}$ & 2965 & 2964 & - & 0.03 \\
\hline NN $(73,299,305,147,129,233 . .445)$ & - & 2968 & - & - & 0.05 \\
\hline Cinnamyl (E)-isoferulate, mono-TMS & $\mathrm{N} / \mathrm{A}$ & 2980 & 2975 & - & 0.56 \\
\hline Cinnamyl (E)-ferulate, mono-TMS & $\mathrm{N} / \mathrm{A}$ & 2990 & 2997 & 0.09 & - \\
\hline NN $(356,341,75,135)$ & - & 2995 & - & 0.26 & - \\
\hline$\beta$-Isomaltose, octa-TMS & $\mathrm{N} / \mathrm{A}$ & 3005 & 3005 & - & 0.05 \\
\hline 3,5,7-Trihydroxy-4'-mathoxyflavone, tri-TMS & $\mathrm{N} / \mathrm{A}$ & 3015 & 3015 & 0.31 & - \\
\hline Pinobanksin 3-hexanoate, di-TMS & $\mathrm{N} / \mathrm{A}$ & 3037 & 3032 & - & 0.04 \\
\hline Cinnamyl (E)-caffeate, di-TMS & N/A & 3044 & 3043 & 0.41 & 0.96 \\
\hline Kaempheride, tri-TMS & $\mathrm{N} / \mathrm{A}$ & 3052 & 3050 & 0.33 & 0.02 \\
\hline 9-Hentriacontene & - & 3076 & 3075 & 0.14 & - \\
\hline Kaempherol, tri-TMS & $\mathrm{N} / \mathrm{A}$ & 3082 & 3078 & 0.29 & 0.04 \\
\hline NN $(414,399)$ & - & 3086 & - & 0.41 & 0.03 \\
\hline NN $(444,401,73,429)$ & - & 3096 & - & 0.18 & 0.02 \\
\hline 3',4',7-Trihydroxyisoflavone, tri-TMS & $\mathrm{N} / \mathrm{A}$ & 3101 & 3098 & 0.14 & 0.09 \\
\hline
\end{tabular}

* trace - below $0.01 \%$ of the total ion current. ** N/A - not available 


\begin{tabular}{|c|c|c|c|c|c|}
\hline Components & CAS & $\mathrm{I}_{\mathrm{T}}^{\operatorname{Exp}}$ & $\mathrm{I}_{\mathrm{T}}^{\mathrm{Lit}}$ & $\begin{array}{l}\text { PPE } \\
\text { [\%] }\end{array}$ & $\begin{array}{l}\text { MPE } \\
\text { [\%] }\end{array}$ \\
\hline Kaempherol, tetra-TMS & $\mathrm{N} / \mathrm{A}$ & 3114 & 3114 & 0.38 & 0.41 \\
\hline NN $(341,73,103,143 \ldots 475,515)$ & - & 3121 & - & - & 0.05 \\
\hline 5,7,4'-Trimethyl-3-methoxyflavone, tri-TMS & $\mathrm{N} / \mathrm{A}$ & 3141 & 3139 & - & 0.09 \\
\hline Apigenin, tri-TMS & $\mathrm{N} / \mathrm{A}$ & 3163 & 3161 & - & 0.09 \\
\hline Triterpenoid $(189,73,129,143,305)$ & - & 3180 & - & - & 0.08 \\
\hline Quercetine, penta-TMS & $4067-66-7$ & 3218 & 3213 & 0.11 & - \\
\hline Isorhamnetin, tetra-TMS & $\mathrm{N} / \mathrm{A}$ & 3245 & 3245 & - & 0.22 \\
\hline p-Coumatate or ferulate, TMS $(219,205,249)$ & $\mathrm{N} / \mathrm{A}$ & 3249 & - & 0.21 & - \\
\hline NN $(73,271,301,103,129,147 \ldots 451,531)$ & - & 3259 & - & - & 0.11 \\
\hline 7-Tritriacontene & $\mathrm{N} / \mathrm{A}$ & 3283 & 3282 & 0.22 & - \\
\hline Myricetin, hexa-TMS & $\mathrm{N} / \mathrm{A}$ & 3303 & 3303 & - & 0.04 \\
\hline Triterpenoid, TMS $(73,189,271,375,129,143)$ & - & 3311 & - & 0.28 & 0.14 \\
\hline NN $(73,301,299,461)$ & - & 3436 & - & - & 0.11 \\
\hline Triterpenoid, TMS $(189,73)$ & - & 3497 & - & - & 0.11 \\
\hline NN $(393,207,73,134,129)$ & - & 3574 & - & - & 0.22 \\
\hline 1,3-Di-p-coumaroyl glycerol, tri-TMS & $\mathrm{N} / \mathrm{A}$ & 3869 & 3869 & 0.02 & - \\
\hline 2-Acetyl-1,3-di-p-coumaroyl glycerol, di-RMS & $\mathrm{N} / \mathrm{A}$ & 3963 & 3963 & 0.89 & - \\
\hline Total & & & & 100.00 & 100.00 \\
\hline
\end{tabular}


Table 2

Group composition of ethanolic extracts from Poland (PPE) and New Zealand (MPE) propolis.

\begin{tabular}{|lll|}
\hline Group of compounds & PPE [\%] & MPE [\%] \\
\hline Flavonoids and chalcones & 49.4 & 52.1 \\
\hline Aromatic acids & 18.3 & 7.8 \\
\hline Cinnamic acid esters & 19.8 & 14.5 \\
\hline Phenylpropenoid glicerydes & 1.3 & 0.0 \\
\hline Aliphatic and aromatic alcohol & 0.2 & 0.8 \\
\hline Aliphatic acids & 0.8 & 0.2 \\
\hline Carbohydrates & 6.2 & 18.7 \\
\hline Sesquiterpenoids & 0.0 & 0.2 \\
\hline Other compounds & 4.0 & 5.7 \\
\hline Total & 100.0 & 100.0 \\
\hline
\end{tabular}

Table 3

Total phenolic content of ethanolic extract from Poland (PPE) and New Zealand (MPE) propolis.

\begin{tabular}{|lll|}
\hline Lp. & Extracts & $\begin{array}{l}\text { TPC }[\mathrm{mg} \text { GAE/g] } \\
\text { Mean } \pm \text { SD }\end{array}$ \\
\hline 1. & PPE & $243.7 \pm 9.0$ \\
\hline 2. & MPE & $245.6 \pm 5.9$ \\
\hline
\end{tabular}

\section{Cytotoxicity and antiproliferative activity}

Chemical compounds present in propolis offer powerful bioactive protection against pathogens and are therefore used by bees to immunise the hive environment [15]. For this reason, they may also serve as a significant source of bioactive substances for pharmaceutical purposes. A number of research studies have focused on the potential utilisation of propolis phenolic compounds in the development of new anticancer drugs $[16,17]$. Our previous study revealed that Polish propolis has strong cytotoxic and antiproliferative activity and, additionally, cooperates with (TMZ) synergistically, enhancing its growthinhibiting activity against glioblastoma U87MG cell line through the reduction of NF-KB activity [8]. In this study, cytotoxicity and antiproliferative activity was determined using DASC cell line derived from a patient and T98G and LN-18 cell lines from ATCC. Dose and time-dependent decreases in DASC viability 
were observed after 24, 48 and $72 \mathrm{~h}$ of incubation with both PPE and MPE (compared to the control) (Fig. 1), and were comparable for both propolis. For DASC cell line we observed a significant reduction in cell numbers $(p<0.05)$ in all concentrations after 24,48 and $72 \mathrm{~h}$; for the dose $30 \mu \mathrm{g} / \mathrm{mL}$, it was $77.9 \pm$ $4.3 \%$ and $81.3 \pm 4.0 \%$ after $24 h, 58.6 \pm 0.3 \%$ and $63.4 \pm 7.8 \%$ after $48 h$, and $47.0 \pm 3.2 \%$ and $51.6 \pm 8.1 \%$ after $72 \mathrm{~h}$ for PPE and MPE, respectively (Fig. 1A,B,C). A significant, but lower than $10 \%$, difference $(\mathrm{p}<$ 0.05 ) in the reduction of DASC cells treated with PPE in comparison to those treated with MPE was observed for the $100 \mu \mathrm{g} / \mathrm{mL}$ concentration after $48 \mathrm{~h}$ (approximately 7\%) (Fig. 1B) and for 20, 50, $100 \mu \mathrm{g} / \mathrm{mL}$ concentrations after $72 \mathrm{~h}(8.4 \%, 6.9 \%, 3.0 \%$, respectively) (Fig. 1C). For T98G cell line we observed a stronger significant reduction in cell numbers $(p<0.05)$, in all concentrations after 24,48 and $72 \mathrm{~h}$ than DASC cell line; for the dose $30 \mu \mathrm{g} / \mathrm{mL}$, it was $78.4 \pm 3.0 \%$ and $75.2 \pm 2.3 \%$ after $24 \mathrm{~h}, 62.8 \pm 1.3 \%$ and $50.8 \pm 7.2 \%$ after $48 \mathrm{~h}$, and $30.7 \pm 7.7 \%$ and $22.0 \pm 8.3 \%$ after $72 \mathrm{~h}$ for PPE and MPE, respectively (Fig. 1D,E,F). Interestingly, dose-dependent decreases in T98G cells viability were observed after 24, 48 and $72 \mathrm{~h}$ but only for the $10-50 \mu \mathrm{g} / \mathrm{mL}$ dose range. After treatment $100 \mu \mathrm{g} / \mathrm{mL}$ dose we observed "reflection effect" because decrease viability was smaller than for $50 \mu \mathrm{g} / \mathrm{mL}$ dose. A significant, difference $(p<0.05)$ in the reduction of T98G cells treated with PPE in comparison to those treated with MPE was observed for the $50 \mu \mathrm{g} / \mathrm{mL}$ concentration after $24 \mathrm{~h}$ (Fig. 1D), for 10, 20,30, $50 \mu \mathrm{g} / \mathrm{mL}$ concentration after $48 \mathrm{~h}$ (Fig. 1E) and for $20,50,100 \mu \mathrm{g} / \mathrm{mL}$ concentrations after $72 \mathrm{~h} \mathrm{(Fig.} \mathrm{1F).} \mathrm{For} \mathrm{LN-18}$ cell line we observed a significant reduction in cell numbers $(p<0.05)$ in all concentrations $20-$ $100 \mu \mathrm{g} / \mathrm{mL}$ after 24,48 and $72 \mathrm{~h}$. For the dose $30 \mu \mathrm{g} / \mathrm{mL}, 81.6 \pm 3.3 \%, 83,2 \pm 0,9 \% 24 \mathrm{~h}, 49.1 \pm 7.8,65.7 \pm$ 8.0 after 48 h, $40.8 \pm 2.5,41.1 \pm 2.9$ respectively PPE and MPE. A significant, difference $(p<0.05)$ in the reduction of $\mathrm{LN}-18$ cells treated with PPE in comparison to those treated with MPE was observed for the $10,30,50,100 \mu \mathrm{g} / \mathrm{mL}$ concentration after $48 \mathrm{~h}$ (Fig. $1 \mathrm{H}$ ), for, 50 and $100 \mu \mathrm{g} / \mathrm{mL}$ concentration after $48 \mathrm{~h}$ (Fig. 1I). Interestingly, significantly stronger cytotoxic effect on LN-18 cells was observed after treatment with PPE than MPE.

The impact of PPE and MPE on DNA biosynthesis in the $\left[{ }^{3} \mathrm{H}\right]$-thymidine incorporation assay was examined in order to confirm if the inhibition of cell viability was caused by a reduction in proliferation capacity. For DASC cell line we found that both PPE and MPE significantly inhibited proliferation - by approximately $10.2 \%$ and $13.2 \%$ after $48 \mathrm{~h}$ and by approximately $23.1 \%$ and $18.6 \%$ after $72 \mathrm{~h}$, respectively (Fig. 2A,B,C). For T98G cell line we observed a significant reduction in proliferation capacity $(p<0.05)$ only for MPE, it was $18.4 \%$ after $24 \mathrm{~h}, 18.6 \%$ after $48 \mathrm{~h}$ and $39.6 \%$ after $72 \mathrm{~h}$ (Fig. 2D,E,F). For LN-18 cell line we found a significant reduction in proliferation capacity $(p<0.05)$ in both, PPE and MPE after 24,48 and $72 \mathrm{~h}$, approximately $40.6 \%$ and $44.5 \%$ after $24 \mathrm{~h}, 39.4 \%$ and $43.3 \%$ after $48 \mathrm{~h}$ and $67.6 \%$ and $75.6 \%$ after $72 \mathrm{~h}$, respectively (Fig. $2 \mathrm{G}, \mathrm{H}, \mathrm{I})$.

Figure. 2. $\left[{ }^{3} \mathrm{H}\right]$-thymidine incorporation into DASC, T98G and $\mathrm{LN}-18$ cells after treatment with PPE and MPE. Legend: [ $\left.{ }^{3} \mathrm{H}\right]$-thymidine incorporation into DASC (A,B,C) and T98G (D,E,F) and LN-18 (G,H,I) cells after $24,48,72 \mathrm{~h}$ of incubation with PPE and MPE (in concentrations $30 \mu \mathrm{g} / \mathrm{mL}$ ). The results are presented as a percentage of control. All statistical analyses were performed using Student-t test (significant changes: ${ }^{*} \mathrm{p}<0.05$ vs control). 
Comparing the effect of both propolis on different glioma cell lines, we found a strong cytotoxic effect against DASC, T98G and LN-18 cells. According to our results, both PPE and MPE have a significant antiproliferative effect on DASC and LN-18 cell lines, while on T98G only MPE. It is worth noting that the cytotoxic and antiproliferative effect of PPE and MPE on DASC cells derived from patient was significant enough to suggest that it is a promising agent for use in supporting anticancer therapy.

Due to the presence of a large number of active substances, propolis exhibits powerful anticancer activity, which has been confirmed in many studies [16-18]. Catchpole et al. [1] demonstrated strong antiproliferative activity of propolis from New Zealand against DLD-1, HCT-116, KYSE-30, NCI-N87 gastrointestinal cancer cells, associated with high levels of phenolic compounds (pinocembrin, pinobanksin-30 -acetate and others). Propolis from Brazil has been demonstrated to exert a strong inhibitory effect on cell growth in glioblastoma (U251 and U343) and fibroblast cell lines (MRC5), but had no effect on apoptosis, demonstrating a cytostatic action [19]. Many publications have explored significant anticancer properties of individual components of propolis. Szliszka and Krol [20] have suggested that polyphenols from propolis sensitise tumor cells to TRAIL-induced apoptosis. The compounds, in combination with TRAIL, exhibit a strong cytotoxic effect on cancer cells [21, 22]. CAPE inhibits NF-kB and enhances the extrinsic pathway of apoptosis in cancer cells induced by TRAIL and Fas receptor stimulation [23]. The most recent research has demonstrated that CAPE displays significant cytotoxicity towards two glioma cell lines Hs683 and LN319 [24]. Other authors have also confirmed that CAPE exhibits powerful antitumor effects on the following cancer cells: fibroblasts from oral submucous fibrosis (OSF), neck metastasis of Gingiva carcinoma (GNM) and tongue squamous cell carcinoma (TSCCa) [25]. Chrysin shows antiproliferative activity against human colorectal cancer cell line HCT-116, liver cancer cell line HepG2 and nasopharyngeal line CNE-1 to TNF-a induced apoptosis and HCT-116, HepG2, cervical cancer cell line HeLa and CNE-1 to TRAIL induced apoptosis [26]. Chrysin induces apoptosis in cancer cells by the activation of caspases, suppression of anti-apoptotic proteins such as IAP, c-FLIP, PI3K/Akt signal pathway, inhibition of IKK and NF-kB activity [27]. In our previous study we demonstrated that natural bee products such as bee bread, royal jelly and honey extract showed varied activity against U87MG and SVGp12 cell lines. Furthermore, the use of these bee products may increase the cytotoxic effect of TMZ on U87MG and SVGp12 cell lines. We also observed that U87MG cells were sensitive to natural bee products, but no impact of natural bee products on DASC cells was noted [10].

\section{Conclusions}

Summarising, these results are the first to show that propolis from Poland and propolis from New Zealand have a strong cytotoxic and antiproliferative effect on Human Diffuse Astrocytoma cell line (DASC) (Grade II glioma) derived from a patient and glioblastoma multiforme T98G and LN-18 cell lines from ATCC. This activity may be associated with the high content of polyphenolic compounds in both propolis. The chemical composition of both propolis was comparable, with marginal differences in the amount of some compounds. These findings suggest that Polish and New Zealand propolis shows promising anticancer activity in the treatment of glioblastoma. However, further studies are required. 


\section{Abbreviations}

PPE

Propolis from Poland

MPE

Propolis from New Zealand

ATCC

American Type Culture Collection

CAPE

Caffeic acid phenethyl ester

DMEM

Dulbecco's modified eagle medium

MEM

Minimal essential medium eagle

FBS

Fetal bovine serum

PBS

Phosphate buffered saline

BSTFA

Bis(trimethylsilyl)trifluoroacetamide )

MTT

Methylthiazolyl diphenyl-tetrazolium bromide

DMSO

Dimethyl sulfoxide

TMS

Trimethylsilyl

GC-MS

Gass chromatography-mass spectrometry

MSD

Mass selective detector

EIMS

Electron ionization mass spectra

$\mathrm{I}_{\mathrm{T}}^{\mathrm{Lit}}$

Linear programmed retention indices

RIExp

Experimental retention indices

TPC

Total phenolic content

FC

Folin-Ciocalteu colorimetric method 
GAE

Gallic acid equivalent

SD

Standard deviation

TMZ

Temozolomide

NF-KB

Nuclear factor kappa-light-chain-enhancer of activated B cells

TRAIL

TNF-related apoptosis-inducing ligand

IAP

Inhibitors of apoptosis proteins

C-FLIP

FLICE-like inhibitory protein

PI3K

Phosphoinositide 3-kinase

Akt

Protein kinase B

IKK

IKB kinase

\section{Declarations}

\section{Authors' contributions}

J.M. and S.K.N. were responsible for conception, study design, obtaining funds, laboratory analysis, statistical analysis and writing the manuscript. V.I. was responsible for the GC-MS laboratory analysis and writing the manuscript. R.M.Ż. and K.J.G.K was responsible for performing laboratory analysis. M.H.B. was responsible for management of the study and was responsible for revising the manuscript critically for important intellectual content. The final manuscript was revised by all co-authors.

\section{Funding}

This study was financially supported by Medical University of Bialystok in project numbered N/ST/ZB/16/004/2216 and N/ST/ZB/17/005/2216. The scientific team was responsible for all stages of the study (design, methodology, analysis, interpretation of the data and writing the manuscript).

\section{Availability of data and materials}

The datasets used and/or analysed during the current study are available from the corresponding author on reasonable request. 


\section{Ethics approval and consent to participate}

The study was approved by the Local Ethical Committee (R-I-002/346/2008).

\section{Conflicts of Interest}

The authors declare no conflict of interest.

\section{Consent for publication}

Not applicable.

\section{References}

1. Catchpole O, Mitchell K, Bloor S, Davis P, Suddes A. Antiproliferative activity of New Zealand propolis and phenolic compounds vs human colorectal adenocarcinoma cells. 2015;106:167-74.

2. Markiewicz-Żukowska R, Car H, Naliwajko SK, Sawicka D, Szynaka B, Chyczewski L, Isidorov V, Borawska MH. Ethanolic extract of propolis, chrysin, CAPE inhibit human astroglia cells. Adv Med Sci. 2012;57:208-16.

3. Isidorov VA. Szczepaniak L, Bakier S. Rapid GC/MS determination of botanical precursors of Eurasian propolis. Food Chem. 2014;142:101-6.

4. Demir S, Aliyazicioglu Y, Turan I, Misir, S, Mentese A, Yaman SO, Akbulut K, Kilinc K, Deger O. Antiproliferative and proapoptotic activity of Turkish propolis on human lung cancer cell line. Nutr Cancer. 2016;68(1):165-72.

5. Aru B, Güzelmeric E, Akgül A, Demirel GY, Kırmızıbekmez H. Antiproliferative activity of chemically characterized propolis from Turkey and its mechanisms of action. Chem Biodivers. 2019;16(7):e1900189.

6. Salim El, Abd El-Magid AD, Farara KM, Maria DS. Antitumoral and antioxidant potential of Egyptian propolis against the PC3 prostate cancer cell line. Asian Pac J Cancer Prev. 2015;16:7641-51.

7. Borawska MH, Naliwajko SK, Moskwa J, Markiewicz-Żukowska R, Puścion-Jakubik A, Soroczyńska J. Anti-proliferative and anti-migration effects of Polish propolis combined with Hypericum perforatum L. on glioblastoma multiforme cell line U87MG. BMC Complement Altern Med. 2016;16:367.

8. Markiewicz-Żukowska R, Borawska MH, Fiedorowicz A, Naliwajko SK, Sawicka D, Car H. Propolis changes the anticancer activity of temozolomide in U87MG human glioblastoma cell line. BMC Complement Altern Med. 2013;13:50.

9. Isidorov VA. Identification of biologically and environmentally significant organic compounds: Mass spectra and retention indices library of trimethylsilyl derivatives. 1rd ed.; PWN: Warszawa, Poland, 2015. 
10. Borawska MH, Markiewicz-Żukowska R, Naliwajko SK, Moskwa J, Bartosiuk E, Socha K, Surażyński A, Kochanowicz J, Mariak Z. The interaction of bee products with temozolomide in human diffuse astrocytoma, glioblastoma multiforme and astroglia cell lines. Nutr Cancer. 2014;66(7):1247-56.

11. Popova M, Giannopoulou E, Skalicka-Woźniak K, Graikou K, Widelski J, Bankova V, Kalofonos H, Sivolapenko G, Gaweł-Bęben K, Antosiewicz B, Chinou I. Characterization and biological evaluation of propolis from Poland. Molecules. 2017;22(7):E1159.

12. Wieczynska A, Wezgowiec J, Wieckiewicz W, Czarny A, Kulbacka J, Nowakowska D, Gancarz R, Wilk KA. Antimicrobial activity, cytotoxicity and total phenolic content of different extracts of propolis from the west pomeranian region in Poland. Acta Pol Pharm. 2017;74(2):715-22.

13. Gemiarto AT, Ninyio NN, Lee SW, Logis J, Fatima A, Chan EW, Lim CS. Isoprenyl caffeate, a major compound in manuka propolis, is a quorum-sensing inhibitor in Chromobacterium violaceum. Antonie Van Leeuwenhoek. 2015;108(2):491-504.

14. Kumazawa S, Hamasaka T, Nakayama T. Antioxidant activity of propolis of various geographic origins. Food Chem. 2004;84:329- 39.

15. Mauricio J, Bankova V. Propolis: Is there a potential for the development of new drugs? J Ethnopharmacol. 2011;133:253-60.

16. Sforcin JM. Biological properties and therapeutic applications of propolis. Phytother Res. 2016;905:894-905.

17. Patel S. Emerging adjuvant therapy for cancer: Propolis and its constituents. J Diet Suppl. 2015;13:1-24.

18. Watanabe MAE, Amarante MK, Conti BJ, Sforcin JM. Cytotoxic constituents of propolis inducing anticancer effects: A review. J Pharm Pharmacol. 2011;63:1378-86.

19. Borges KS, Brassesco MS, Scrideli CA, Soares AE, Tone LG. Antiproliferative effects of Tubi-bee propolis in glioblastoma cell lines. Genet Mol Biol. 2011;34:310-14.

20. Szliszka E, Krol W. Polyphenols isolated from propolis augment TRAlL-induced apoptosis in cancer cells. Evid Based Complement Alternat Med. 2013;2013:731940.

21. Bernardi S, Secchiero P, Zauli G. State of art and recent developments of anti-cancer strategies based on TRAIL. Recent Pat Anticancer Drug Discov. 2012;7:207-17.

22. Li X, Huang Q, Ong CN, Yang XF, Shen HM. Chrysin sensitizes tumor necrosis factor-a-induced apoptosis in human tumor cells via suppression of nuclear factor-kappaB. Cancer Lett. 2010;293(1):109-16.

23. Szliszka E, Czuba ZP, Domino M, Mazur B, Zydowicz G, Krol W. Ethanolic extract of propolis (EEP) enhances the apoptosis-inducing potential of TRAIL in cancer cells. Molecules. 2009;14:738-54.

24. Morin P, St-Coeur PD, Doiron JA, Cormier M, Poitras JJ, Surette ME, Touaibia M. Substituted caffeic and ferulic acid phenethyl esters: Synthesis, leukotrienes biosynthesis inhibition, and cytotoxic activity. Molecules. 2017;22(7): E1124. 
25. Lee YJ, Liao PH, Chen WK, Yang CC. Preferential cytotoxicity of caffeic acid phenethyl ester analogues on oral cancer cells. Cancer Lett. 2000; 153:51-6.

26. Li X, Wang JN, Huang JM, Xiong XK, Chen MF, Ong CN, Shen HM, Yang XF. Chrysin promotes tumor necrosis factor (TNF)-related apoptosis-inducing ligand (TRAIL) induced apoptosis in human cancer cell lines. Toxicol in Vitro. 2011;25(3):630-5.

27. Sawicka D, Car H, Borawska MH, Nikliński J. The anticancer activity of propolis. Folia Histochem Cytobiol. 2012;50(1):25-37.

\section{Figures}

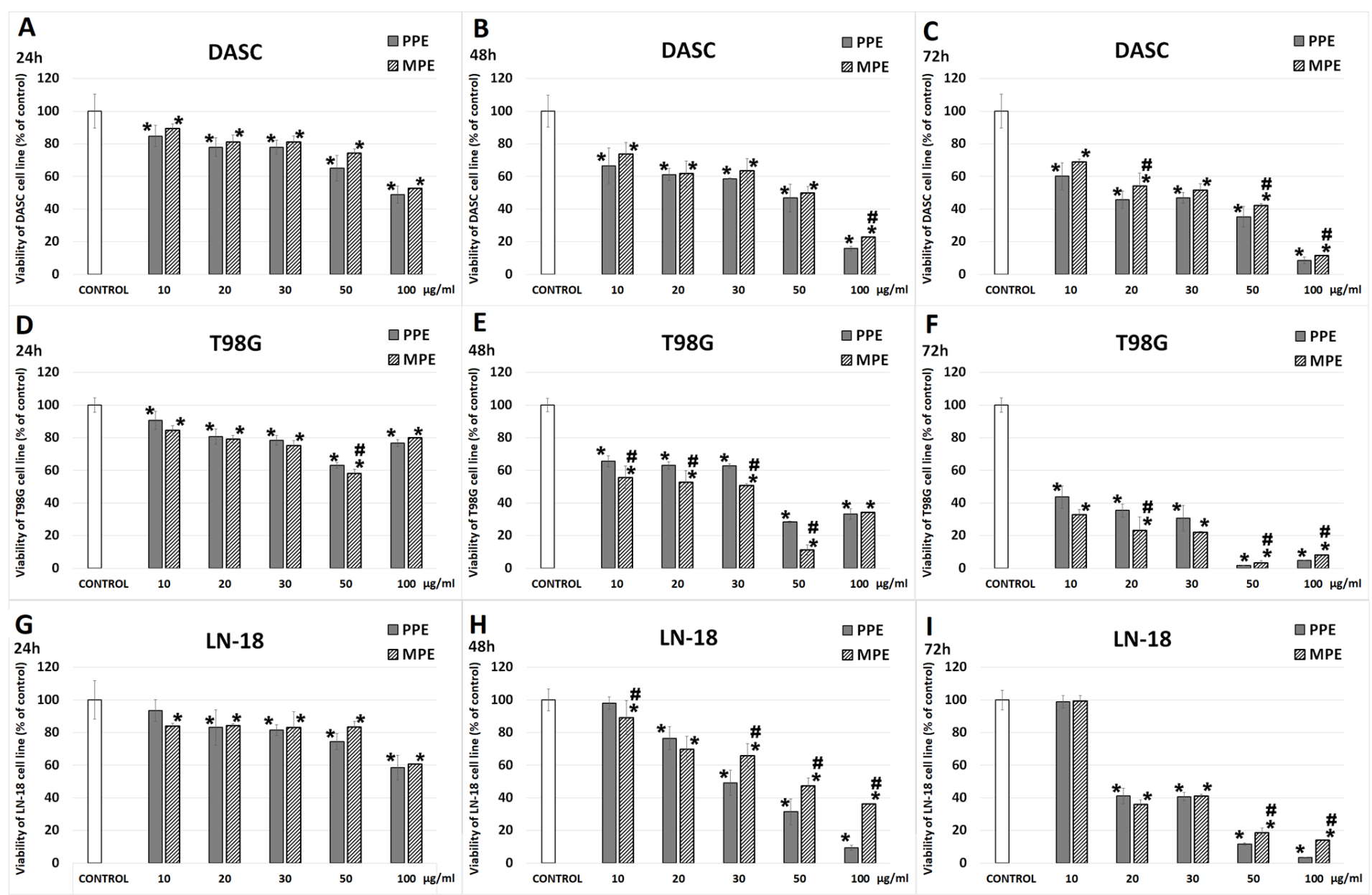

\section{Figure 1}

Viability of DASC, T98G and LN-18 cells after treatment with PPE and MPE. Legend: Cytotoxicity effect of PPE and MPE (in concentrations 10, 20, 30, 50, $100 \mu \mathrm{g} / \mathrm{mL}$ ) of DASC (A,B,C), T98G (D,E,F) and LN-18 $(\mathrm{G}, \mathrm{H}, \mathrm{I})$ cells after 24,48 and $72 \mathrm{~h}$ of incubation. The results are presented as a percentage of control. All statistical analyses were performed using Student-t or U Manna-Whitneya tests (significant changes: ${ }^{*} \mathrm{p}<0.05$ vs control, \# PPE vs MPE). 


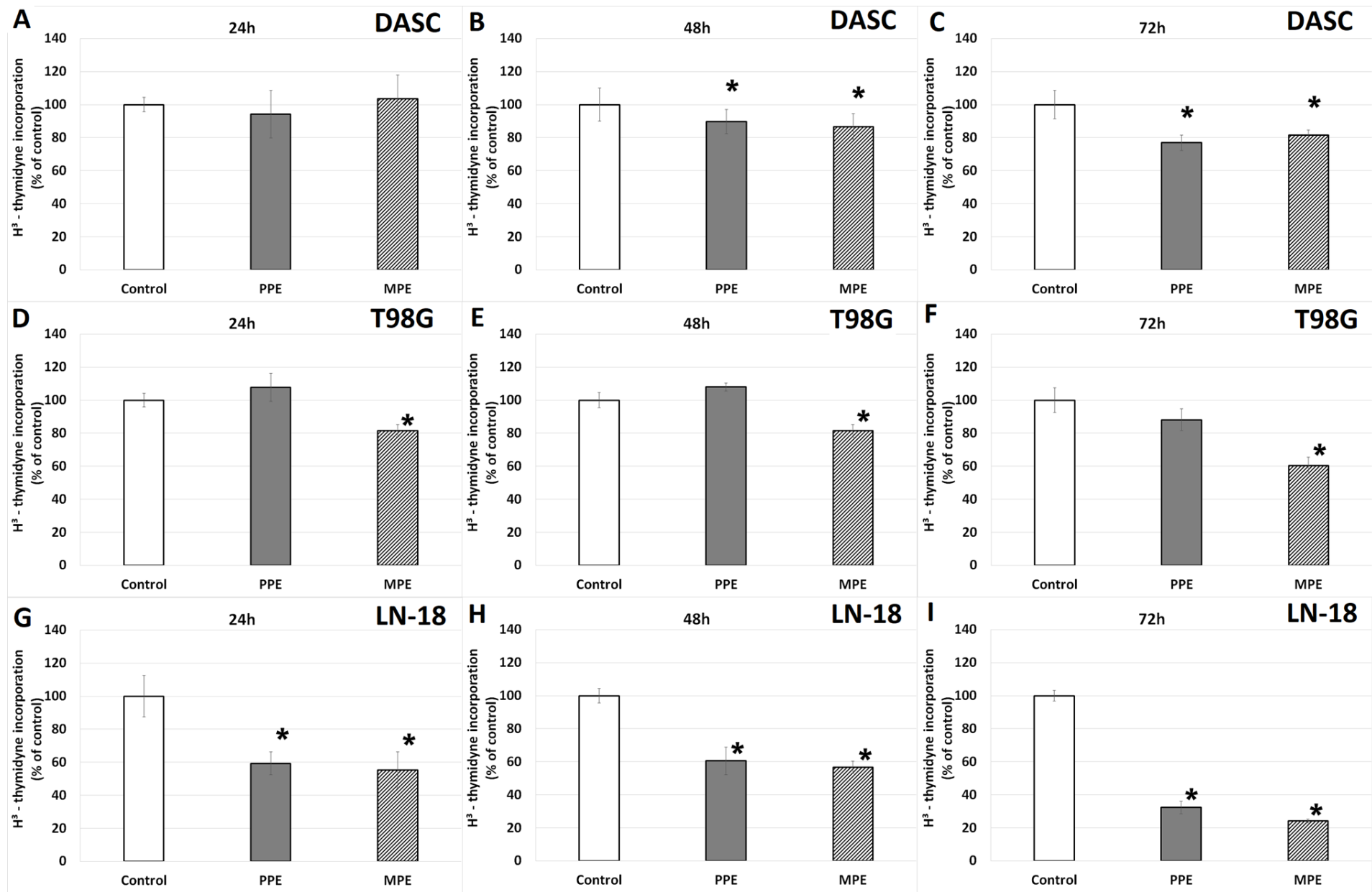

Figure 2

[3H]-thymidine incorporation into DASC, T98G and LN-18 cells after treatment with PPE and MPE.

Legend: [3H]-thymidine incorporation into DASC $(A, B, C)$ and T98G $(D, E, F)$ and LN-18 $(G, H, I)$ cells after 24, 48, $72 \mathrm{~h}$ of incubation with PPE and MPE (in concentrations $30 \mu \mathrm{g} / \mathrm{mL}$ ). The results are presented as a percentage of control. All statistical analyses were performed using Student-t test (significant changes: ${ }^{*} \mathrm{p}<0.05$ vs control). 